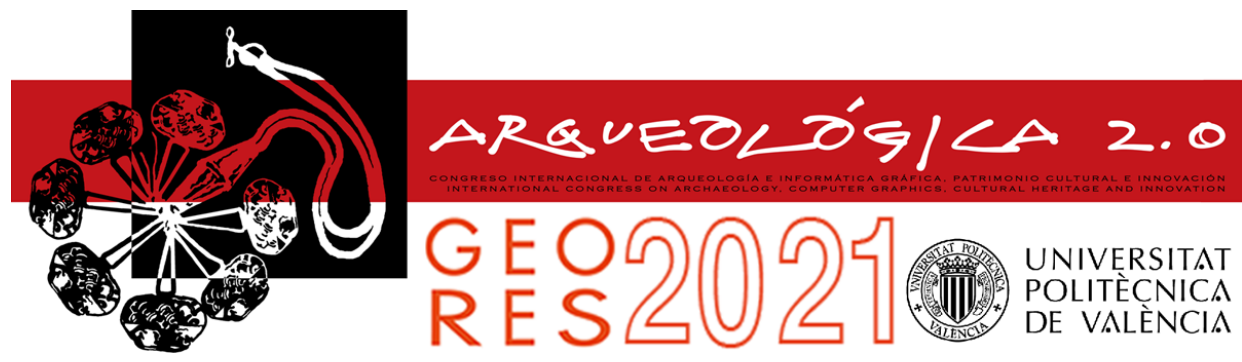

Proceedings of the joint international event $9^{\text {th }}$ ARQUEOLÓGICA

$2.0 \& 3^{\text {rd }}$ GEORES

Valencia (Spain).

26-28 April 2021

\title{
TLS AND IMAGE-BASED ACQUISITION GEOMETRY FOR EVALUATING SURFACE CHARACTERIZATION
}

\author{
Giacomo Patrucco*, Stefano Perri, Antonia Spanò \\ Department of Architecture and Design (DAD), Politecnico di Torino, Viale Mattioli 39, 10125 Torino, Italy. giacomo.patrucco@polito.it; \\ stefano.perri@polito.it; antonia.spano@polito.it
}

\begin{abstract}
:
In the framework of cultural heritage documentation projects, it is very frequent the use of dense and detailed 3D models derived from range-based or image-based techniques. These reality-based models represent an effective and powerful solution to document the geometries, the surfaces and the characteristic of heritage assets. In fact, these technologies allow us to accurately describe the level of detail and the surface characterization of the materials, and also to provide precious support for the evaluation of the conservation status of the surveyed structures. In particular, the object studied in the presented research experience is the Morano sul Po arch (Morano sul Po, AL, Piedmont, Italy), a valuable example of an industrial archaeology asset. For the knowledge process of the arch, both LiDAR systems and photogrammetric strategies have been used, in order to properly document the geometry, the consistency of the material and the decays. The specific aim of the research presented in this paper is the evaluation of the advantages and the critical issues of using a telescopic pneumatic pole to raise the position of the scans from the ground and decrease the angle of incidence of the laser beam on the surveyed object. For this reason, the study also takes into consideration the use of mini UAVs and their flexibility to effectively acquire vertical surfaces even at elevated heights, comparing the density and the roughness of the derived model with the data obtainable by using TLS systems.
\end{abstract}

Keywords: TLS, UAV photogrammetry, surface characterisation, multi-sensor, cultural heritage, 3D model

\section{Introduction}

The survey techniques aimed at cultural heritage documentation and the generation of dense and accurate 3D models have undergone an impressive evolution in the last few years. Nowadays, thanks to the availability of many tools, methods and strategies it is possible to effectively respond to very diversified needs connected to heritage documentation processes (Murtiyoso, Grussenmeyer, Suwardhi \& Awalludin, 2018; Chiabrando, Sammartano, Spanò \& Spreafico, 2019).

The use of topographic systems allows the developing of the entire workflow and the generation of the 3D models in a common reference system. In addition, it also provides valuable instruments to properly perform an accuracy check on the final results.

Among the most widespread trends in the field of multisensor techniques aimed at obtaining multi-scale and multi-resolution models, rapid mapping techniques are usually preferred, allowing rapid site occupation and increasing the survey sustainability.

Moreover, it should be underlined that advantages are evident in terms of efficiency and effectiveness derived from the automation of image-based techniques, the development of co-registration techniques as well as the
ICP algorithms - allowing to align point clouds of different nature and resolution. Due to these significant developments, the possibility to perform productive fusions and integrations among data from different sources and techniques is being strengthened in the heritage documentation sector. (Muñumer \& Lerma 2015; Ramos \& Remondino 2015; Georgopoulos et al., 2017; Güleç Korumaz, 2019).

Considering the aforementioned technological advances, the obtainable 3D models can be more and more addressed to users-oriented purposes, ensuring centimeter and sometimes even millimeter accuracies, depending on the purposes for which the 3D models are made. The aforementioned technologies and achievable results can provide valid support to the maintenance, conservation and restoration interventions planning, contributing to define materials decay or structural anomalies and pathologies. In the framework of dissemination and communication of the heritage, it is necessary to consider virtual applications or augmented reality navigation, moreover, replicas generations and the realisation of virtual museums can be achieved using these methods and strategies (Balletti \& Ballarin, 2019; Barbieri, Bruno \& Muzzupappa, 2018). 
In this contribution, the main aim is to highlight how the acquisition geometry - not only as regards the image collection for photogrammetric purposes, but also considering the TLS (Terrestrial Laser Scanning) sampling - can affect the accuracy of the 3D model and the possibility to properly document the characterisation and the roughness of the surveyed surfaces.

In the next sections, the effectiveness of a device able to change the point of view of the acquisition of the scans will be evaluated. Furthermore, it will be investigated how to ration the acquisition of the point clouds by modifying the distance between the TLS system and the object of interest during point clouds collection and the inclination of the laser beam with respect to the surveyed surfaces.

The results obtained are also compared with an imagebased 3D model, obtained through acquisitions performed using a mini UAV (Unmanned Aerial Vehicle) system. Nowadays this kind of technology seems particularly promising for documenting the heritage, since it allows to acquire hard to reach surfaces with a relatively high level of detail. In fact, it should be underlined that, due to the versatility and manageability of these systems, the acquisitions can be performed at very short distances from the surfaces of interest. (Adami et al. 2019; Xiang, Xia \& Zhang, 2019).

Since its inception, laser scanning technology has been represented as an object of interest for the community of measurements science and surveyors. The extraordinary potential of this technology has been immediately highlighted and the calibration issues of the various sensors and measurement systems (both time-of-flight and phase-shift scanners) have been deeply investigated (Boehler, Bordas Vicent, \& Marbs, 2003).

Many researchers operating in the fields of 3D survey and modelling of the built heritage focused their studies on this topic, and perhaps one of the most recognized advantages was the possibility of deriving true digital orthophotos from Dense Digital Surface Models (DDSM) (Balletti, Guerra, Lingua \& Rinaudo, 2003). That laid the foundations for close collaborative applications of both TLS and image-based techniques that characterize many cultural heritage documentation projects, ranging from the most important sites of cultural interest to artistic or archaeological heritage.

The quality assessment of TLS systems has been investigated (Vögtle, Schwab \& Landes, 2008), considering the influence of different materials in the measurements of the range of terrestrial scanners. In addition, intensity analyses have been considered. Other authors have studied the different results deriving from the direction of the laser beam intercepting the surface, and how the distance between the laser scanner and the surveyed object affect the quality of the acquired point cloud (Tucci, Bonora \& Guardini, 2013). Generally, these issues can be interpreted with calibration matters (Lichti \& Licht, 2006).

In the framework of point cloud acquisition, high accuracies and high level of detail are often required. In some cases, the shape of the surveyed object is not the only feature to be investigated, but also the surface characterization. For this reason, in order to study the effects that the distance and the incidence angle of the laser beam produce on the surfaces (featuring the local accuracy and noise of the point cloud), a comparison among 3D models derived from different scans planned positions have been investigated in the current paper.

The possibility to acquire scans by a device that raises the acquisition centre of the scanner from the ground has been also tested.

Tripods or telescopic pole are sometimes used in cultural heritage documentation, usually equipped with a camera, and as observed in various researches, the possible instability issues can be considered not significant (Bitelli, Dellapasqua, Girelli, Sanchini \& Tini, 2017).

In this framework, extension arms, telescopic poles and lift platforms are often used and they certainly require great attention even when the scale of the survey is the architectural one (Corso, Casals, Marco, López \& Garcia Almirall, 2018), but they need further assessments if the characterization of roughness materials is also considered and investigated, as proposed in the next sections.

\section{Case Study: the Morano sul Po arch}

The Morano sul Po arch (Morano sul Po, AL, Piedmont, Italy) (Fig. 1a) was built in the years of the economic boom, after World War II. It plays a symbolic role as a demonstration of the construction possibilities achieved with the experiments of structural engineering carried out in the 1930s and with the innovative materials produced by the cement industry. The arch was also functional to the production cycle as it allowed the passage of the raw material from the Coniolo quarry, on the other side of the Po river, to the Morano furnace. In fact, it protected the Trino-Morano-Casale provincial road from possible losses of material from the trolleys transported by the cableway (Fig. 1b). Now the arch has been subjected to a conservation project aimed at the dissemination of the socio-economic and cultural values coming from the cement industry together with its innovations in the constructive systems.

\section{Methodology}

\subsection{Instrumentation and data acquisition}

In December 2019, a 3D metric acquisition campaign has been carried out by a team from G4CH Lab of Politecnico di Torino with the aim of documenting the conservation status of the paraboloid arch of Morano in the framework of the planning of the aforementioned scheduled restoration works. A complete LiDAR survey and a series of photogrammetric acquisitions (both terrestrial and from UAV) have been performed in order to provide a multisensor three-dimensional documentation of the considered structure belonging to the valuable industrial heritage assets of the areas adjacent to Casale Monferrato, where the cement production activities have always played a key-role in the framework of the local economy.

As regards the TLS survey, 19 LiDAR point clouds have been acquired using 2 laser scanners: a Faro Focus ${ }^{3 D} X$ 330 and a Faro Focus ${ }^{3 D}$ S120 (Fig. 2). The acquisition scheme of the collected scans can be observed in Figure 3 . Since the main specifications of the two employed devices - which are reported in Table 1 - are equal, except for the operational acquisition range, the point 
clouds from both the laser scanners can be considered comparable in terms of density and noise.
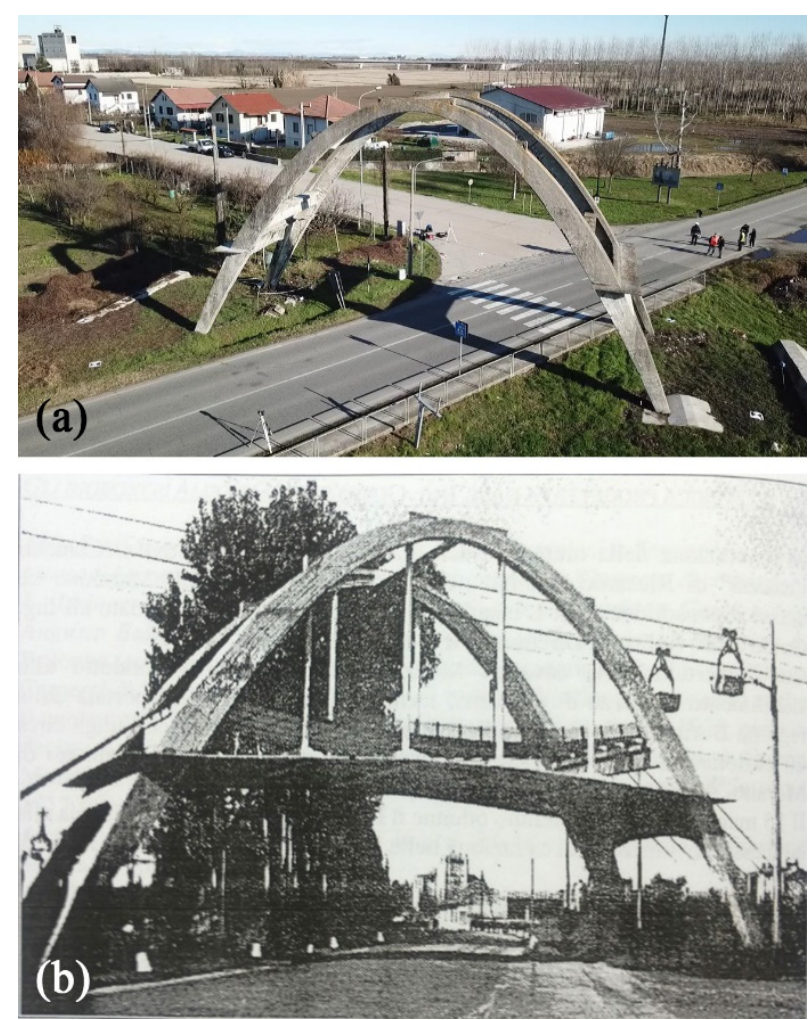

Figure 1: The paraboloid arch of Morano sul Po: a) Current image; b) Historical image (1955) of the arch, during the transport operations of raw material over the Trino-MoranoCasale provincial road.

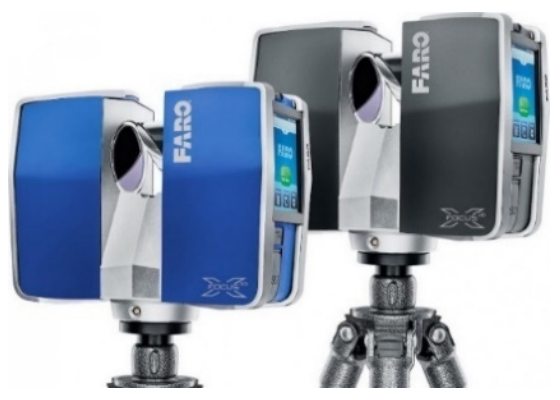

Figure 2: On the left, Faro Focus ${ }^{3 D} \times 330$. On the right, Faro Focus $^{3 \mathrm{D}} \mathrm{S} 120$.

Table 1: Main specifications of the used laser scanners (Faro Focus $^{3 D} \times 330$ and Faro Focus ${ }^{3 D}$ S120).

\begin{tabular}{c|c|c} 
Laser scanner & $\begin{array}{c}\text { Faro Focus } \\
330\end{array}$ & $\begin{array}{c}\text { Faro Focus } \\
\text { S120 }\end{array}$ \\
\hline $\begin{array}{c}\text { Distance Range } \\
\text { Horizontal and } \\
\text { vertical range }\end{array}$ & $0.6-330 \mathrm{~m}$ & $0.6-120 \mathrm{~m}$ \\
$\begin{array}{c}\text { Distance accuracy } \\
\text { Acquisition speed }\end{array}$ & $\begin{array}{c} \pm 2 \mathrm{~mm} @ 10 \mathrm{~m} \\
\text { up to } 976.000 \mathrm{pt} / \mathrm{s}\end{array}$ & $\begin{array}{c} \pm 2 \mathrm{~mm} @ 10 \mathrm{~m} \\
\text { up to } 976.000 \mathrm{pt} / \mathrm{s}\end{array}$
\end{tabular}

The TLS acquisition has been planned in order to homogeneously cover the maximum number of surfaces of the Arch, with the aim of providing an adequate overlapping between the adjacent scans to facilitate the successive registration operations. As concerns the resolution of the collected point clouds, the acquisitions have been performed using a resolution of $1 \mathrm{pt} / 6 \mathrm{~mm}$ at $10 \mathrm{~m}$ of distance.

On this occasion a pneumatic telescopic pole has been tested in order to raise the laser scanner and acquire a point cloud of the higher areas of the arch with a lower angle of incidence of the laser signal - the angle between the laser beam and the normal direction of the surveyed surface (Soudarissanane, Lindenbergh, Menenti \& Teunissen, 2009; Soudarissanane, Lindenbergh, Menenti \& Teunissen, 2011; Roca-Pardiñas, Argüelles-Fraga, de Asís López \& Ordóñez) - in comparison to the scans acquired from the ground level.

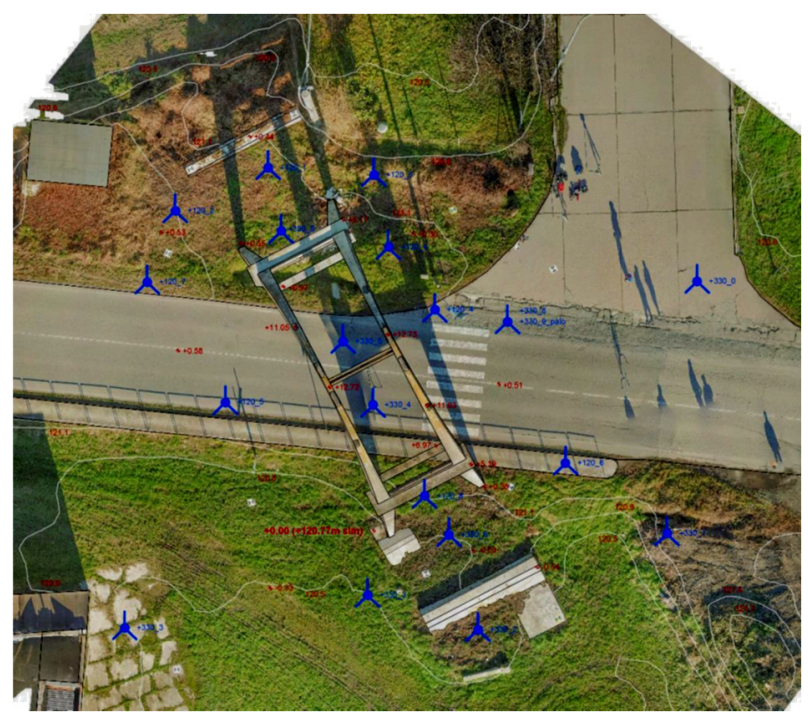

Figure 3: Position of the acquired scans.

These last aspects represent a key issue in the framework of heritage building documentation: in order to provide complete metric documentation of heritage buildings especially as regards the particularly high facades and the outdoor areas - there is often the need to acquire data from an elevated perspective.

As noted above, this is the reason why, in the last few years it was possible to observe an incremental increase of the use of UAV systems in many fields and disciplines - including heritage assets documentation (Bakirman et al., 2020; Chiabrando, Sammartano, Spanò \& Teppati, 2020) - for aerial monitoring and survey purposes. The flexibility, the efficiency and the rapidity of these kinds of solutions are the reasons why in recent years the use of drones has become a very popular and effective strategy to collect images from hard-to-reach perspectives, with a particular focus on the mini UAV solutions compliant to the needs and purposes of this research.

Also in the case presented a photogrammetric aerial survey has been carried out. The UAV system used for the aerial acquisitions is a commercial drone developed from DJI (model: DJI Mavic Pro, equipped with a digital camera FC220) (Fig. 4). The principal specifications of the employed drone can be observed in Table 2. Several flights have been performed with both nadir and oblique camera configuration (to effectively acquire the higher surfaces and the extrados of the Arch) and a planned overlapping between the images $\geq 80 \%$. In total 384 images (resolution $4000 \times 3000$ pixels) have been collected during these flights (mean shooting distance $\approx$ 
15-20 m) with a mean GSD (Ground Sample Distance) of $\approx 5 \mathrm{~mm}$.

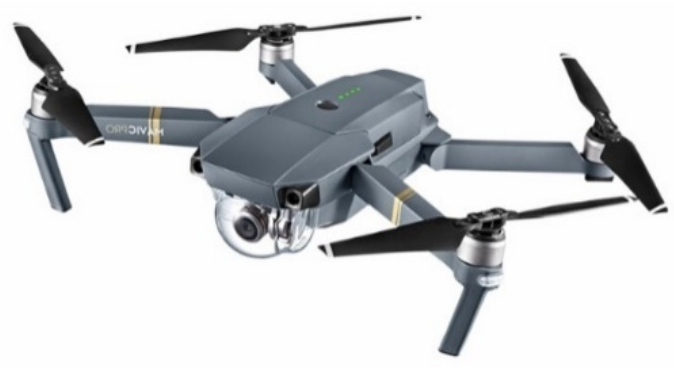

Figure 4: DJI Mavic Pro.

Table 2: Main specifications of DJI Mavic Pro.

\begin{tabular}{c|c} 
DJI Mavic Pro (FC220) & Camera specifications \\
\hline Sensor & CMOS 1/2.3" \\
Effective pixels & 12.35 Mpixels \\
Lens & FOV $78.8^{\circ} 5 \mathrm{~mm} \mathrm{f} / 2.2(26 \mathrm{~mm}$ \\
in $35 \mathrm{~mm}$ format equivalent $)$ \\
ISO Range & $100-1600$ \\
Shutter Speed & $8 \mathrm{~s}-1 / 8000 \mathrm{~s}$ \\
Max image size & $4000 \times 3000$ pixels \\
Photo format & JPG, DNG
\end{tabular}

In addition, 222 images (resolution: $8688 \times 5791$ pixels) have been collected using a DSLR (Digital Single Lens Reflex) camera (model: Canon EOS 5DSR) equipped with a $25 \mathrm{~mm}$ Zeiss lens (principal specifications in Table 3 ). In this case, the aim was to cover the lower areas of the concrete structure, in order to achieve a complete photogrammetric model of the Arch of Morano by integrating the UAV acquired images with the terrestrial ones.

Table 3: Main specifications of Canon EOS 5DSR.

\begin{tabular}{c|c} 
Canon EOS 5DSR & Camera specifications \\
\hline Sensor & CMOS 50.3 Mpixels \\
Sensor size & $36 \times 24 \mathrm{~mm}$ \\
Image size & $8688 \times 5792$ pixels \\
Lens & Zeiss ZE $/$ ZF.2 \\
Focal lenght & Distagon T*25 mm/ $2 / 2$ \\
& $25 \mathrm{~mm}$
\end{tabular}

With the aim of registering all the data acquired during the campaign in a common reference system, 34 topographic points have been measured (both artificial checkboard target and natural points) from two vertices, using a total station. The coordinates of the two vertices have been derived from a control network adjustment; measures were carried out using two GNSS receivers (static technique) in order to georeference the data derived from the survey.

\subsection{Data processing}

The 19 LiDAR scans have been processed using the Faro Scene software, following a consolidated registration strategy which consists of a preliminary alignment of the point clouds using an ICP (Iterative Closest Point) algorithm, and in a subsequent rigid rototraslation of the block composed by the registered scans using the coordinates of the measured markers. At the end of this procedure the observed accuracy on the control points is $\approx 6 \mathrm{~mm}$ (this value represents the mean $3 \mathrm{D}$ deviation between the coordinates observed on the target in the point cloud and the reference coordinates) (Lachat, Landes \& Grussenmeyer, 2018) (Fig. 5).

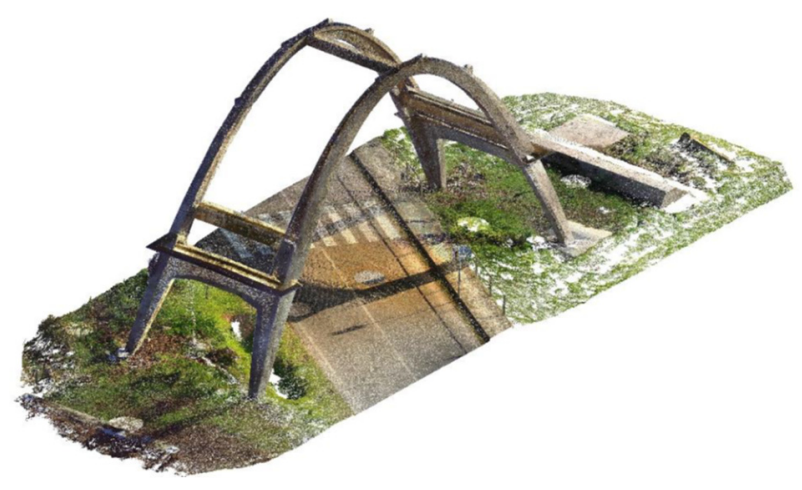

Figure 5: Lidar point cloud.

As regards the image-based approach, the 606 images acquired from both the UAV system and the DLSR camera have been processed (Figs. 6 and 7) using the SfM-based (Structure-from-Motion) software Agisoft Metashape, following the standard pipeline (images masking; relative orientation of the images and tie-points extraction; georeferencing and bundle adjustment using the acquired Ground Control Points; accuracy evaluation using both control points and check points; dense point cloud generation) (Ewertowski, Tomczyk, Evans, Roberts, \& Ewertowski, 2019). As concerns both the image alignment phase and the generation of the dense point cloud, the quality parameter has been set on "high" (which means that all the images have been processed at their original resolution without downscaling).

Of the 34 points measured with the total station, 28 have been used as GCPs (Ground Control Points) and 6 as CPs (Control Points) in order to perform an accuracy check on the final results. The RMSE (Root-Mean-Square Error) after the bundle block adjustment is reported in Table 4

Table 4: Main specifications of Canon EOS 5DSR.

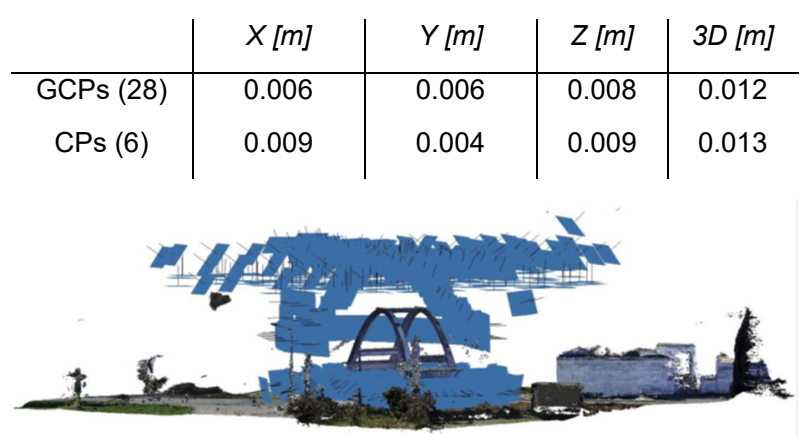

Figure 6: Image alignment and tie points generation. 


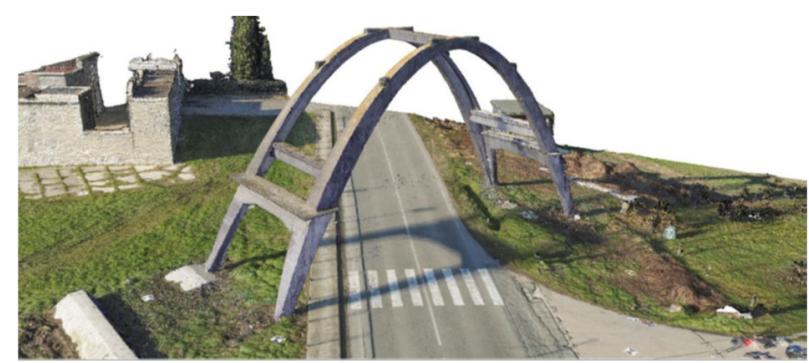

Figure 7: Photogrammetric point cloud.

\section{Results analysis}

As stated in the previous section, in this framework the use of a pneumatic telescopic pole to raise the laser scanner above the ground level (and therefore to acquire points on the elevated surfaces of the Arch façade with a low incidence angle) has been tested (Fig. 8).

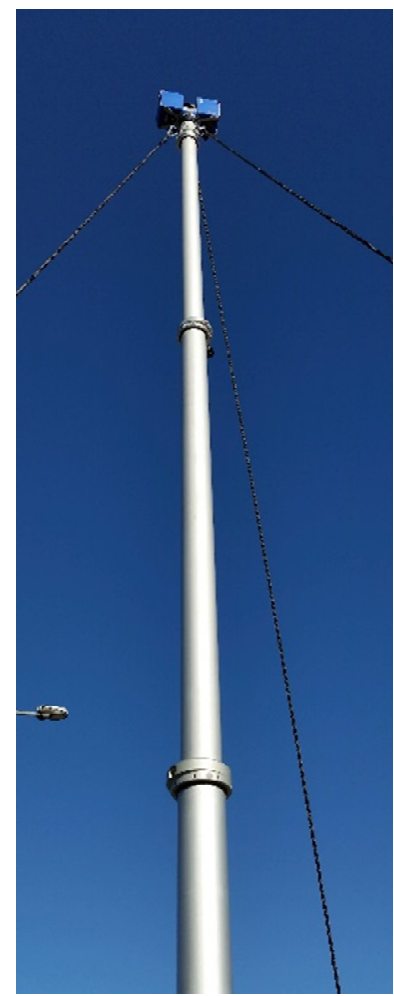

Figure 8: Telescopic pole equipping the Faro laser scanner during the case study 3D survey.

A set of focused analysis has been performed on the upper area of the east façade of the Arch, to compare both the scans acquired at different heights (thanks to the telescopic pole) and different distances.

For this comparison, among the 19 scans acquired during the TLS survey to achieve the complete model of the Arch, four have been selected according to the acquisition geometry (Fig. 9):

- 1 scan (Scan A) acquired raising the laser scanner by using a telescopic pneumatic pole. The acquisition distance is approximately $10 \mathrm{~m}$. The scan has been performed in calm wind conditions.
- 1 scan (Scan B) acquired at ground level. The acquisition distance is approximately the same of Scan A.

- 1 scan (Scan C) acquired at ground level. The acquisition distance is approximately $25 \mathrm{~m}$.

- 1 scan (Scan D) acquired very close to the Arch.
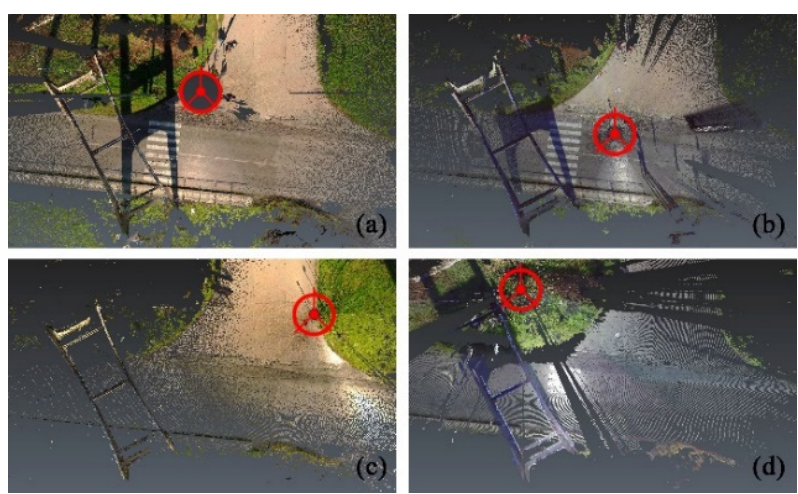

Figure 9: Acquisition scheme of each of the considered LiDAR scan (the position of the laser scanner is indicated by the red symbol): a) scanner on the pneumatic telescopic pole, acquisition from medium distance; b) scanner on ground level, acquisition from medium distance; c) scanner on ground level, acquisition from long distance); d) scanner on ground level, acquisition from short distance.

The photogrammetric point cloud has been considered too in the framework of these analyses, since - as reported in the previous sections - the UAV photogrammetry has proved itself as a very competitive and viable alternative. In this case, starting from the oriented block of 606 images, a dense point cloud has been generated enabling only the images collected with the camera in a forward camera configuration (Fig. 10).

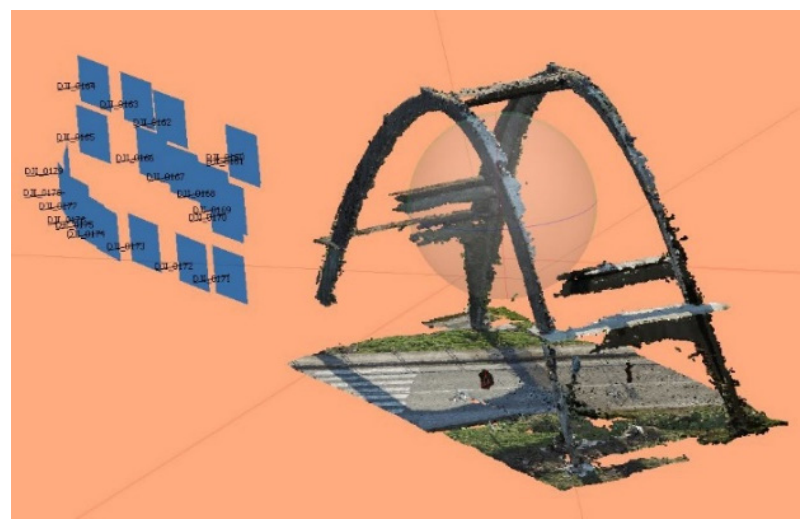

Figure 10: Photogrammetric point cloud generated from the images acquired with a forward camera asset.

For the analyses described in the following paragraphs, a portion located in the highest part of the arch façade has been considered (Fig. 11). Firstly, a density analysis has been carried out using the CloudCompare open-source platform (Fig. 12). For each point the number of neighbours has been estimated considering a sphere with a radius of $5 \mathrm{~cm}\left(\mathrm{n}^{\circ}\right.$ points/sphere $\left.r=5 \mathrm{~cm}\right)$. As predictable, the point cloud characterised by a higher density is the one acquired from the telescopic pole (Scan A) due to the lower distance of acquisition to the surveyed surface (in comparison to Scan B, where the scanner was placed at a greater distance, and Scan C, acquired from 
the ground level) and to the lower inclination of the laser signal. In fact, in the case of Scan D, the laser scanner is positioned very close to the Arch, but due to the very high incidence angle of the laser beam the density of the acquired point cloud is extremely low (a mean value of 30 pt./V. sphere $r=0.05 \mathrm{~cm}$ ).

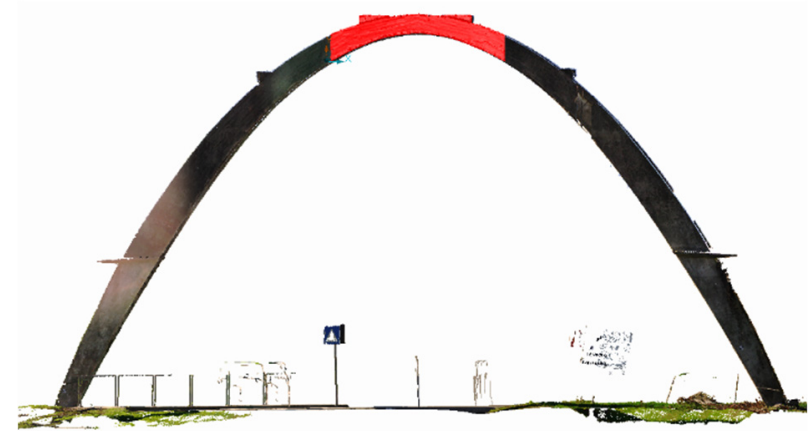

Figure 11: Portion of the arch (red points) considered for the analyses which have been carried out during this research.

However, as evidenced from the subsequent roughness analysis, the surface of the telescopic pole point cloud is affected by a topological error due to the vibrations transmitted from the laser scanner to the pole during the acquisition of the scan. The CloudCompare roughness analysis allows to evidence the points that deviate from the best fitting plane (computed between the neighbouring points in a sphere that in this case has been set with a radius of $5 \mathrm{~cm}$ ). In this case, the observed mean value is comparable for each of the considered point clouds, but as regards Scan $A$ the regular corrugation observable along its surface (Fig. 13a) begins to become more evident.

This last characteristics in extremely evident observing the mesh triangulated from each point cloud (Fig. 14). However, besides the corrugation issue, it is possible to notice that the mesh computed from Scan A is (as expected) the one with a higher resolution and level of detail. The only other point cloud showing a high degree of characterization of the surface which allows to adequately describe the consistency of the material of the scanned object, is Scan B; while in Scan C (acquired from a longer distance - $25 \mathrm{~m}$ - to the arch) and in Scan $D$ (characterised by a very high incidence angle of the laser beam to the surface due to the proximity to the concrete structure) it is possible to observe a low level of detail and material consistency interpretation (the two meshes are extremely smoothed).

After the detection of the regular corrugation due to the vibrations of the scanner, an investigation has been performed in order to evaluate how the metric accuracy of the acquired point cloud was affected by this topological error. With the aim of quantifying the dimension of the undulating deformation observed in Scan A, a best-fitting plane has been interpolated on a portion of the considered point cloud and a planarity analysis has been carried out with the 3DReshaper software using the extracted plane as reference. From this analysis, the topologically erroneous corrugation (previously detected on the roughness analysis and on the surface of the triangulated mesh) can be estimated with a maximum deviation of $\pm 1 \mathrm{~mm}$ (Fig. 15).
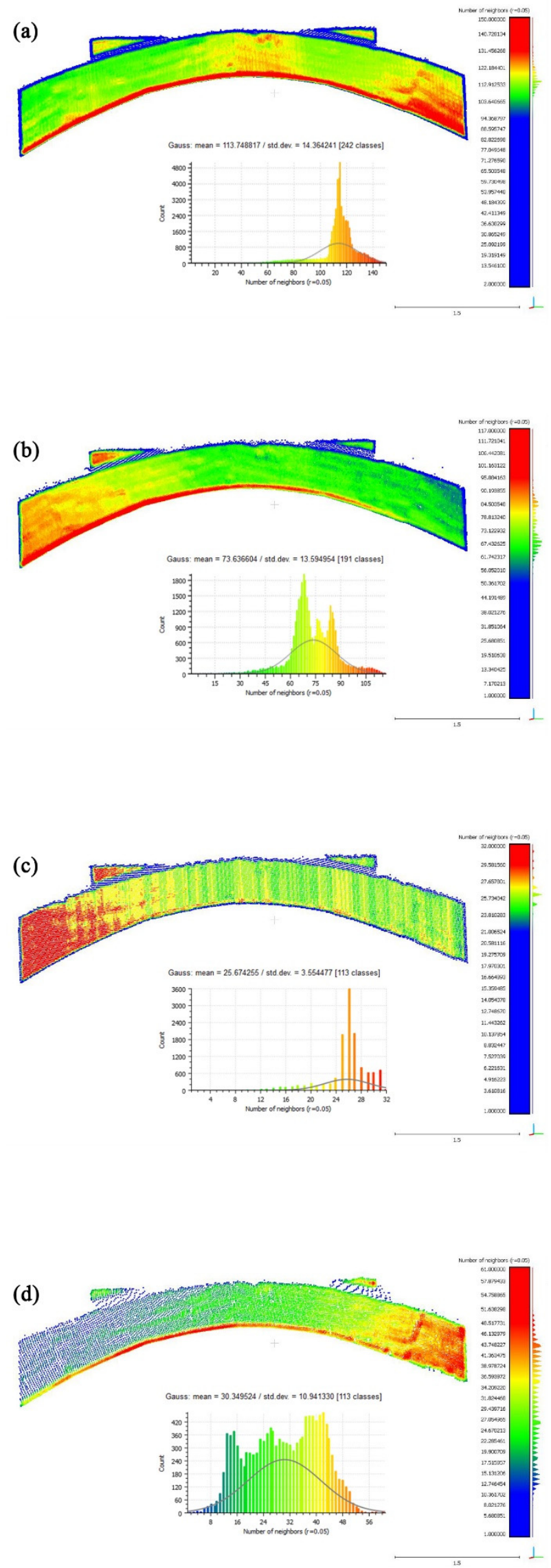

Figure 12: Density analysis of a portion of the upper part of the Arch façade, performed with CloudCompare: a) Scan A; b) Scan B; c) Scan C; d) Scan D. 

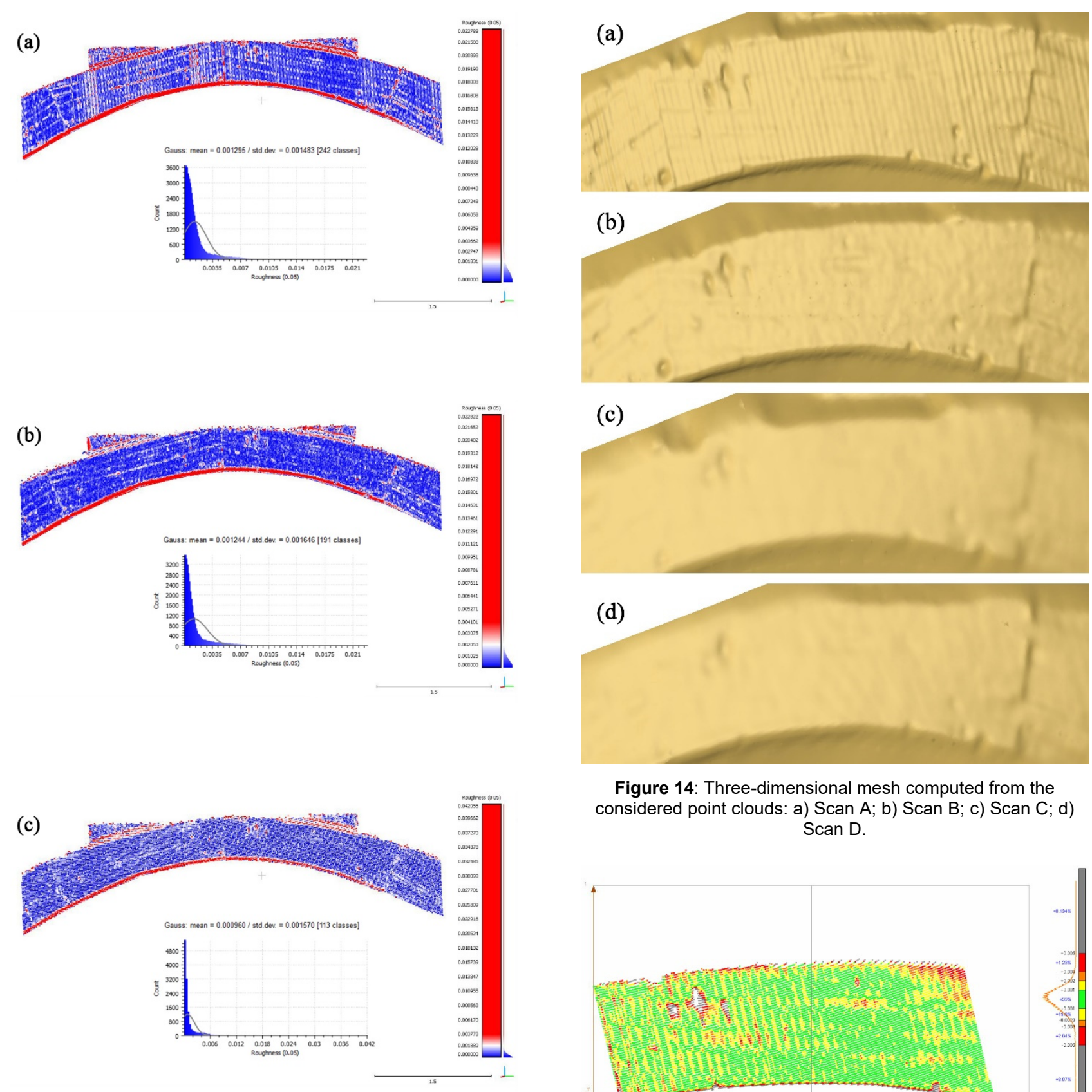

Figure 14: Three-dimensional mesh computed from the considered point clouds: a) Scan A; b) Scan B; c) Scan C; d) Scan D.

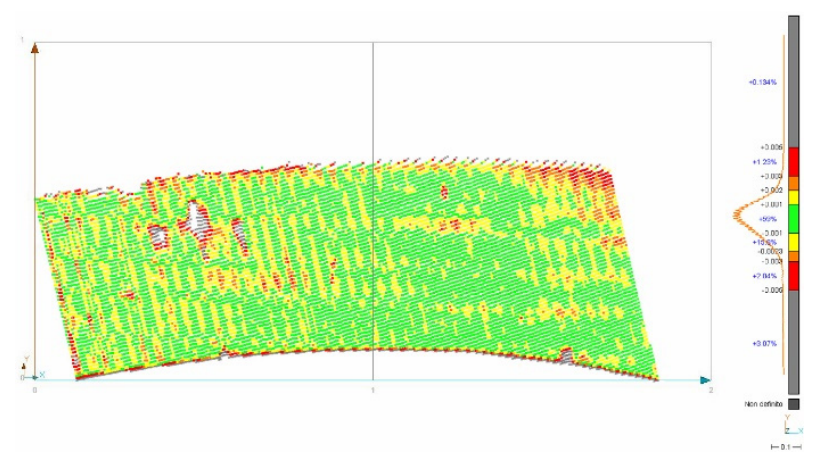

Figure 15: Planarity analysis carried out on a portion of the point cloud acquired from the telescopic pole.

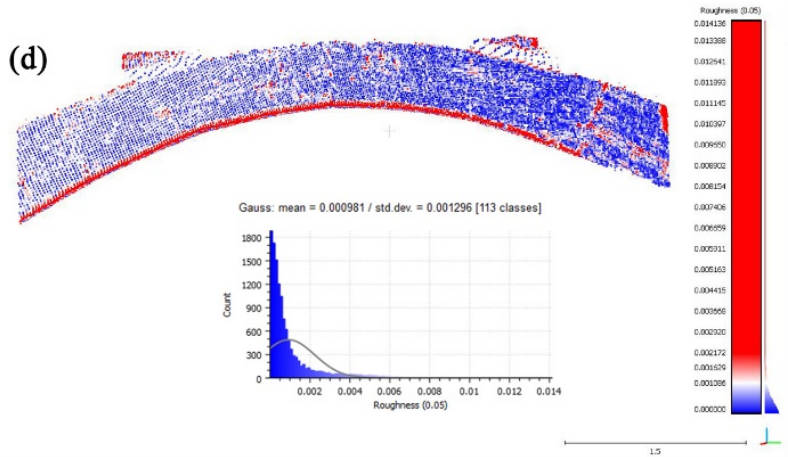

In order to perform a comparison between the products achievable using different strategies (LiDAR techniques or an image-based approach), the same analyses (density analysis; roughness analysis; 3D mesh computation) has been performed on the dense point cloud generated from UAV dataset using an SfM-based photogrammetric pipeline. As it is possible to observe in Figure $16 \mathrm{a}$, the data density is much smaller (mean value $\approx 24$ pt. $/ \mathrm{V}$. sphere $r=0.05 \mathrm{~cm}$ ) in comparison to Scan $A$ and Scan B.

Figure 13: Roughness analysis of a portion of the upper part of the Arch façade, performed with CloudCompare: a) Scan A; b) Scan B; c) Scan C; d) Scan D. 
(a)
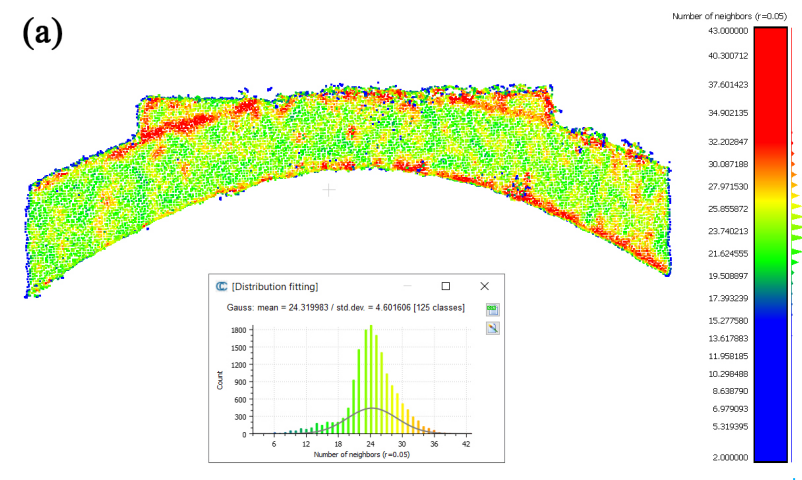

(b)

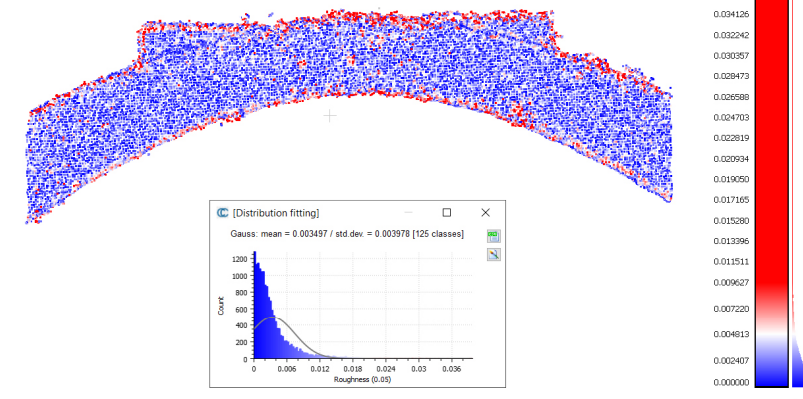

(c)

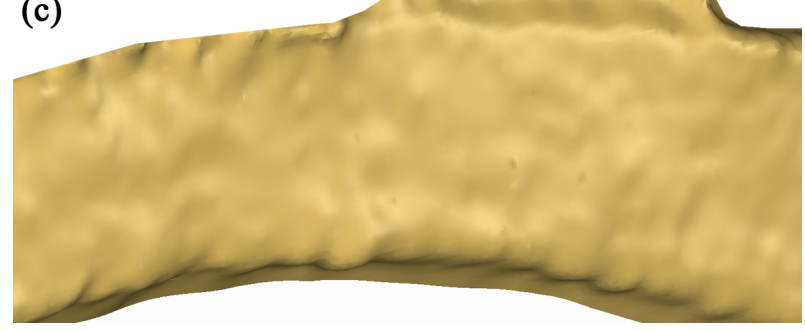

Figure 16: Analysis performed on UAV dataset: a) Density analysis; b) Roughness analysis; c) Triangulated mesh.

Furthermore, in the 3D reconstruction (Fig. 16c) a very high smoothness and a low 3D characterisation in terms of consistency of the material roughness are observable. However, it should be underlined that the image-based approach can provide, besides the geometric information, also the radiometric content of the object, through the production of a texturized 3D model or the generation of orthoimagery. As regards this particular aspect, the photogrammetric image-based approach is very competitive and preferable to the traditional LiDAR survey strategies. In fact, despite nowadays almost all of the commercial laser scanners are equipped with an embedded optical camera - or they have the possibility to equip an external one - the radiometric quality of the data derived from laser scanning technologies in generally lower in comparison to photogrammetric products.

\section{Discussion}

As observed in the previous section, starting from the performed analysis it is possible to confirm how the acquisition geometry during a LiDAR survey - and, in the specific, the position of the laser scanner - influences different aspects of the final point cloud. As expected and as evidenced by the results obtained with Scan B and Scan $C$ it is evident that the density of the data decrease with increasing distance or in correspondence of high incidence angles of the laser beam. Also the spatial resolution and the level of the detail are lower.

As regards this last aspect, the two best performing point clouds are certainly Scan A and Scan B. However, in the case of Scan $A$ the regular pattern corrugation generated from the vibrations and micro-movements transmitted to the pneumatic telescopic pole from the laser scanner rotation represents a heavy limit in the definition of a topological correct characterisation of the surveyed surface. In the framework of the current research experience, and if the aim is to accurately document the roughness of the concrete surface of the arch, Scan B represents the best compromise between density of the data and level of detail. However, it should be underlined that, in the case of a higher structure (the arch is approximately $13 \mathrm{~m}$ ) and/or a smaller space in front of the object of interest, this acquisition strategy it would not be possible.

In terms of completeness, the photogrammetric point cloud derived from mini UAV acquisitions is very competitive. In fact, following a LiDAR approach, even using the telescopic pole it was possible to acquire the surfaces of the extrados: to pursue this goal it would have been necessary to develop a further strategy to increase the acquisition height of the laser scanner (e.g. using a lift platform). However, the level of detail of the photogrammetric is extremely lower in comparison to the elevated surface characterisation observable in the LiDAR data (especially in the scans acquired from a low distance and where the incidence angle of the laser beam is not excessive). In connection to this issue, a solution can be represented by the use of UAV systems characterised by larger dimensions (specifically designed to lift heavier payloads) and equipped with more performing camera sensors. However, it would not be possible to exploit the specific advantages connected to mini UAV solutions (greater manoeuvrability; greater safety during acquisitions; lower cost; etc.).

Another effective solution in order to boost the quality of the final results and to reach a comparable level of detail is related to the shooting distance, which in this case should be drastically decreased. However, this is not always possible since a lower shooting distance corresponds to a greater number of acquired images to properly cover all the surfaces of the surveyed object, at the expenses of the data processing phase and file storage. In the case of the presented research, the acquisition distance has been planned in order to reach a fair compromise between the obtainable resolution of the final 3D model and the management of the data.

However, it must be recognised that thanks to the radiometric contents - and, consequently, metric products as 3D texturized mesh and orthoimagery achievable using the photogrammetric approach - it is nevertheless possible to obtain valuable information about the material and the consistency of the acquired surfaces (on the contrary to LiDAR data where the information is connected to the spatial resolution of the point clouds, since the poor radiometric resolution of the embedded camera). 


\section{Conclusion}

The presented analysis has allowed an evaluation of the advantages and critical issues connected with the use of a device to raise the centre of acquisition of a range scanner from the ground, comparing the achievable points model and derived mesh surface to another one generated by an image-based approach and using a mini UAV.

We can say that the topological description of the roughness and the characterisation of the surface is a very particular goal pursuable during the 3D metric documentation process. The acquisition strategies and the techniques to be used must be defined after an attentive critical analysis of the aims of the survey, considering the scale, the desired level of detail and the characteristics of the object of interest. It is often also necessary to consider the characteristics of the adjacent space, in order to effectively plan the geometry of the acquisitions.

On many occasions - like the one described in the current paper - a multi-sensor and multi-scale approach can provide the necessary flexibility to properly document a cultural heritage asset. In addition, the opportunity offered by the possibility to perform the fusion between data from different sources and to merge different information contents can contribute to reaching a more complete and deeper knowledge of the buildings belonging to our legacy.

\section{Acknowledgements}

The research is connected to a conservation and valorisation project that selected the Morano sul Po arch as the symbol of the cement industry, which provided in the past many innovations and socio-economic development in the area.

We would like to express our gratitude to "Il Cemento" Association for having asked for our cooperation in this cultural project.

Moreover, we would like to thank our colleague Lorenzo Teppati Losè for precious support in data acquisition.

\section{References}

Adami, A., Fregonese, L., Gallo, M., Helder, J., Pepe, M., \& Treccani, D. (2019). Ultra light UAV systems for the metrical documentation of Cultural Heritage: applications for architecture and archaeology. ISPRS - International Archives of the Photogrammetry, Remote Sensing and Spatial Information Sciences, XLII-2/W17, 15-21.

Bakirman, T., Bayram, B., Akpinar, B., Karabulut, M. F., Bayrak, O. C., Yigitoglu, A., \& Seker, D. Z. (2020). Implementation of ultra-light UAV systems for cultural heritage documentation. Journal of Cultural Heritage, 44, 174-184.

Balletti, C., \& Ballarin, M. (2019). An application of integrated 3D technologies for replicas in Cultural Heritage. International Journal of Geo-Information, 8(6), 285.

Balletti, C., Guerra, F., Lingua, A., \& Rinaudo, F. (2003). True digital orthophoto of the San Marco basilica in Venice. ISPRS - International Archives of the Photogrammetry, Remote Sensing and Spatial Information Sciences, XXXIV(5)MV12, 43-48.

Barbieri, L., Bruno, F., \& Muzzupappa, M. (2018). User-centered design of a virtual reality exhibit for archaeological museums. International Journal on Interactive Design and Manufacturing (IJIDeM), 12(2), 561-571.

Bitelli, G., Dellapasqua, M., Girelli, V. A., Sanchini, E., \& Tini, M. (2017). 3D Geomatics Techniques for an Integrated Approach to Cultural Heritage Knowledge: the case of San Michele in Acerboli's church in Santarcangelo di Romagna. ISPRS - International Archives of the Photogrammetry, Remote Sensing and Spatial Information Sciences, XLII-5/W1, 291-296.

Boehler, W., Bordas Vicent, M., \& Marbs, A. (2003). Investigating laser scanner accuracy. ISPRS - International Archives of the Photogrammetry, Remote Sensing and Spatial Information Sciences, XXXIV(5)/W15, 696-701.

Chiabrando, F., Sammartano, G., Spanò, A., Spreafico, A. (2019). Hybrid 3D Models: When Geomatics Innovations Meet Extensive Built Heritage Complexes. ISPRS International Journal of Geo-Information, 8(3), 124.

Chiabrando, F., Sammartano, G., Spanò, A., \& Teppati Losè, L. (2020). Very light UAV data and ranging methods for heritage documentation. In: Barba, S., Parrinello, S., Limongiello, M., Dell'Amico, A. (ed.), D-SITE Drones - Systems of Information on culTural hEritage. For a spatial and social investigation, Pavia University Press, Pavia, Italy, 5869 .

Corso, J., Casals, J., Marco, A., López, D., \& Garcia Almirall, P. (2018). Data taking and quality control for complex surveys: Case study Sagrada Familia. World heritage and knowledge: representation, restauration, redesign, resilience, 960970.

Ewertowski, M. W., Tomczyk, A. M., Evans, D. J. A., Roberts, D. H., \& Ewertowski, W. (2019). Operational Framework for Rapid, Very-high Resolution Mapping of Glacial Geomorphology Using Low-cost Unmanned Aerial Vehicles and Structure-from-Motion Approach. Remote Sensing, 11(1), 65.

Georgopoulos, A., Lambrou, E., Pantazis, G., Agrafiotis, P., Papadaki, A., Kotoula, L., Lampropoulos, K., Delegou, E., Apostolopoulou, M., Alexakis, M., \& Moropoulou, A. (2017). Merging geometric documentation with materials characterization and analysis of the history of the holy aedicule in the church of the holy sepulchre in Jerusalem. ISPRS - International Archives of the Photogrammetry, Remote Sensing and Spatial Information Sciences, XLII$5 / W 1,487-494$. 
Güleç Korumaz, S. A. (2019). Improved documentation of Cultural Heritage using digital photogrammetry with visible and thermal images from Unmanned Aerial Vehicles (UAV). Ph.D. Dissertation, University of Braunschweig - Institute of Technology and University of Florence. Thesis advisors: Niemeier, W., Tucci, G.

Lachat, E., Landes, T., \& Grussenmeyer, P. (2018). Comparison of point cloud registration algorithms for better result assessment - Towards an open-source solution. ISPRS - International Archives of the Photogrammetry, Remote Sensing and Spatial Information Sciences, XLII-2, 551-558.

Lichti, D., \& Licht, M. (2006). Experiences with Terrestrial Laser Scanner Modelling and Accuracy Assessment. ISPRS International Archives of the Photogrammetry, Remote Sensing and Spatial Information Sciences, XXXVI(5), 155160.

Muñumer, E., \& Lerma, J. L. (2015). Fusion of 3D data from different image-based and range-based sources for efficient heritage recording. 2015 Digital Heritage, 1, 83-86.

Murtiyoso, A., Grussenmeyer, P., Suwardhi, D., \& Awalludin, R. (2018). Multi-Scale and Multi-Sensor 3D Documentation of Heritage Complexes in Urban Areas. ISPRS International Journal of Geo-Information, 7(12), 483.

Ramos, M. M., \& Remondino, F. (2015). Data fusion in Cultural Heritage - A Review. ISPRS - International Archives of the Photogrammetry, Remote Sensing and Spatial Information Sciences, XL-5/W7, 359-363.

Roca-Pardiñas, J., Argüelles-Fraga, R., de Asís López, F., \& Ordóñez, C. (2014). Analysis of the influence of range and angle of incidence of terrestrial laser scanning measurements on tunnel inspection. Tunnelling and underground space technology, 43, 133-139.

Soudarissanane, S., Lindenbergh, R., Menenti, M., \& Teunissen, P. (2009). Incidence angle influence on the quality of terrestrial laser scanning points. Proceedings ISPRS Workshop Laserscanning 2009, Paris, France.

Soudarissanane, S., Lindenbergh, R., Menenti, M., \& Teunissen, P. (2011). Scanning geometry: influencing factor on the quality of terrestrial laser scanning points. ISPRS Journal of Photogrammetry and Remote Sensing, 66(4), 389-399.

Tucci, G., Bonora, V., \& Guardini, N. (2013). Analysis of the factors affecting 3D models resolution - Application to the recording of vaults in Sangallo's House. ISPRS - Annals of the Photogrammetry, Remote Sensing and Spatial Information Sciences, II-5/W1, 307-312.

Vögtle, T., Schwab, I., \& Landes, T. (2008). Influences of different materials on the measurements of a terrestrial laser scanner (TLS). ISPRS - International Archives of the Photogrammetry, Remote Sensing and Spatial Information Sciences, Vol. XXXVII-B5, 1061-1066.

Xiang, T. Z., Xia, G. S., \& Zhang, L. (2019). Mini-Unmanned Aerial Vehicle-based Remote Sensing: Techniques, applications, and prospectives. IEEE Geoscience and Remote Sensing Magazine, 7(3), 29-63. 